\section{Prevalência e fatores associados à realização da mamografia na faixa etária de 50-69 anos: um estudo baseado na Pesquisa Nacional por Amostra de Domicílios (2003)}

\author{
Prevalence and factors associated with \\ mammograms in the 50-69-year age group: a \\ study based on the Brazilian National Household \\ Sample Survey (PNAD-2003)
}

Maria Fernanda Lima-Costa 1

Divane Leite Matos 1

\section{${ }_{1}^{1}$ Núcleo de Estudos em Saúde Pública e Envelhecimento, Centro de Pesquisas René Rachou, Fundação Oswaldo Cruz/Universidade Federal de Minas Gerais, Belo Horizonte, Brasil. \\ Correspondência M. F. Lima-Costa Núcleo de Estudos em Saúde Pública e Envelhecimento, Centro de Pesquisas René Rachou, Fundação Oswaldo Cruz/Universidade Federal de Minas Gerais. Av. Augusto de Lima 1715, sala 609, Belo Horizonte, $M G$ 30190-002, Brasil. lima-costa@cpqrr.fiocruz.br}

\section{Abstract}

This study was developed to estimate the prevalence and factors associated with mammograms among Brazilian women, based on data from the National Household Sample Survey (PNAD) in 2003. 16,570 and 10,722 women aged 50-59 and 60-69 years participated in the study. 43\% of participants aged 50-69 years had received a mammogram in the previous two years. The rate was lower among older women (37\%) as compared to the younger group (46\%). In both age groups, having received a mammogram in the previous two years was positively and independently associated with urban residence, region of residence, years of schooling, family income, Papanicolaou exam, number of physician visits, and health plan coverage. The results showed that the prevalence of mammograms in the previous two years is low among older Brazilian women, mainly in the 60-69-year group. The study identified striking inequalities in mammogram coverage. Further research is needed to better understand the reasons for such inequalities and thus help overcome them.

Mammography; Health of the Elderly; Women's Health; Women

\section{Introdução}

O câncer de mama é a principal causa de morte por neoplasias entre adultas brasileiras, sobretudo entre as mais velhas 1,2. As mortes por câncer de mama podem ser reduzidas quando o tumor é descoberto precocemente, sendo a mamografia o instrumento mais efetivo para diagnóstico precoce desse tumor. Entretanto, existe alguma controvérsia com relação às faixas etárias nas quais esse exame deva ser realizado, como parte do exame rotineiro de saúde. Nos Estados Unidos, recomenda-se a realização da mamografia a cada um ou dois anos entre mulheres com 40-69 anos e reconhece-se que ela possa trazer benefícios, mesmo a partir dos 70 anos de idade, quando a incidência de câncer de mama é mais alta (U.S. Preventive Services Task Force. http:/ / www. ahcpr.org, acessado em 13/Out/2005). O documento americano Healthy People 2010 estabelece como meta a realização da mamografia a cada um ou dois anos em $70 \%$ das mulheres com 40 69 anos de idade ${ }^{3}$. Por outro lado, no Canadá, a realização da mamografia, como parte do exame geral de saúde, é recomendada para a faixa etária de 50-69 anos, mas o grupo tarefa canadense ressalta que não existem evidências suficientes para descartar o potencial de prevenção da mamografia na faixa etária de 40-49 anos (Canadian Task Force on Prevention Health Care. http://www. ctfphc.org, acessado em 13/Out/2005). De qualquer forma, existe consenso de que as evidências 
da efetividade da mamografia para a redução da mortalidade por câncer de mama são mais fortes na faixa etária de 50-69 anos de idade.

No Brasil, existe um interesse crescente pela mamografia como instrumento da Saúde Pública. Uma das expressões desse interesse é a inclusão do tema em grandes inquéritos nacionais. Em um inquérito recentemente conduzido em 15 capitais brasileiras, observou-se que existe grande variação na cobertura do exame: na faixa etária de 50-69 anos, a realização da mamografia nos dois anos precedentes variou entre 37 e $77 \% 4$.

Fatores associados à realização da mamografia são ainda pouco conhecidos no Brasil. Na Região Metropolitana de Belo Horizonte, Minas Gerais, Brasil, observou-se que a realização da mamografia há dois anos ou menos entre mulheres com 50-69 anos de idade estava fortemente associada à cobertura por plano privado de saúde (odds ratio - OR = 3,35; intervalo de confiança de 95\% - IC95\%: 2,51-4,48), verificando-se que essa forte associação persistia mesmo após estratificação pela escolaridade 5,6. Na cidade de Pelotas, Estado do Rio Grande do Sul, Brasil, observou-se que a realização da mamografia, em qualquer época da vida, entre mulheres com 40-69 anos, estava positivamente associada à classe social, à história familiar de câncer de mama, ao uso atual de terapia de reposição hormonal, à biópsia/cirurgia prévia de mama, ao exame clínico da mama e à consulta ginecológica 7 .

A Pesquisa Nacional por Amostra de Domicílios (PNAD) incluiu, no seu suplemento de saúde em 2003, informações sobre a realização da mamografia, tornando-se a maior base de dados de base populacional sobre a realização desse exame no Brasil. Essa foi a base de dados utilizada no presente trabalho, que foi conduzido com os seguintes objetivos: (1) estimar a prevalência da realização da mamografia entre mulheres com 50-69 anos de idade; (2) verificar se existe diferença na cobertura do exame entre as faixas etárias de 50-59 e 60-69 anos e; (3) determinar os fatores associados à realização da mamografia em cada uma dessas faixas etárias.

\section{Metodologia}

Pesquisa Nacional por Amostra de Domicílios (PNAD)

A PNAD é conduzida, anualmente, com o objetivo de obter informações sobre características demográficas, habitação, educação, trabalho e rendimentos da população brasileira. A pesquisa tem abrangência nacional, tendo incluído, em 2003, um suplemento com informações relativas a condições de saúde e uso de serviços de saúde, além da realização da mamografia. A coleta de dados foi realizada entre os dias 21 e 27 de setembro de 20038.

A PNAD baseia-se em uma amostra probabilística de domicílios obtida em três estágios de seleção. No primeiro estágio, é feita a seleção dos municípios, que são classificados em auto-representativos, com probabilidade 1 de pertencer à amostra, e em não auto-representativos, com probabilidade de pertencer à amostra proporcional à população residente. No segundo estágio, são selecionados os setores censitários, cuja probabilidade de inclusão é proporcional ao número de domicílios existentes no setor. No terceiro estágio, os domicílios são amostrados em cada setor censitário, sendo investigadas as informações relativas a todos os residentes no domicílio ${ }^{8}$. No presente trabalho, foram analisados os dados relativos às mulheres participantes da amostra da PNAD com idade entre 50 e 69 anos de idade.

\section{Variáveis}

\section{- Variável dependente}

A variável dependente deste trabalho é a realização da mamografia. Na PNAD 2003, a informação sobre a realização do exame foi obtida por meio da seguinte pergunta: "Quando foi a última vez que você fez uma mamografia?”. As respostas foram codificadas em quatro grupos: menos de um ano, um ano a dois anos, três anos ou mais e nunca fez mamografia ${ }^{8}$. No presente trabalho, foram considerados três estratos para a descrição da prevalência (há dois anos ou menos, três anos ou mais e nunca) e dois estratos (dois anos ou menos $v s$. três anos ou mais) para análise dos fatores associados à realização da mamografia.

\section{- Variáveis independentes}

As variáveis independentes deste trabalho incluem: características sócio-demográficas, indicadores das condições de saúde e indicadores de uso de serviços de saúde.

As seguintes características sócio-demográficas foram consideradas no trabalho: condição rural ou urbana do domicílio; macrorregião de residência; número de pessoas residentes no domicílio (morar só ou não); número de anos completos de escolaridade e renda familiar per capita. Os níveis de renda domiciliar per capita dos participantes do estudo foram agrupados em quintis e transformados em salários mínimos da época, quando esse salário correspondia a R\$ 240,00. A seguinte distribuição foi obtida: 
$<\mathrm{R} \$ 120,00$ (quintil inferior); R\$ 120,00-219,00; $\mathrm{R} \$ 220,00-329,00 ; \mathrm{R} \$ 330,00-619,00$ e $\geq \mathrm{R} \$ 620,00$ (quintil superior).

Os indicadores das condições de saúde incluídos neste estudo foram percepção da saúde, número de doenças crônicas e grau de dificuldade para realizar pelo menos uma entre três atividades da vida diária (alimentar-se, tomar banho e/ou ir ao banheiro). Com relação à percepção da saúde, existe alguma controvérsia quanto ao uso da informação fornecida pela PNAD, porque, nesta pesquisa, a proporção de respostas dadas por outra pessoa é muito alta. O questionamento baseia-se na premissa de que a percepção da saúde representa uma visão subjetiva do indivíduo, que não pode ser aferida por outra pessoa. Entretanto, um estudo recente, usando dados da PNAD 1998 e da PNAD 2003, mostrou que o uso de outro respondente não modifica a distribuição da autoavaliação da saúde entre idosos, nem os fatores associados à mesma 9. Dessa forma, no presente trabalho, optou-se por considerar toda a informação relativa a percepção da saúde, desconsiderando-se quem respondeu à entrevista. A informação sobre a presença de doenças crônicas foi obtida por meio da pergunta: "Algum médico ou profissional de saúde já disse que você tinha ...?”. No presente trabalho, o número de doenças crônicas foi calculado somando-se as respostas afirmativas às perguntas sobre as seguintes doenças/condições crônicas: hipertensão, artrite/reumatismo, doença do coração, diabete, asma/bronquite, doença renal crônica, câncer, cirrose, depressão e doença da coluna/dor nas costas.

Os indicadores de uso de serviços de saúde considerados foram: realização de exame Papanicolau durante a vida, adotando-se a estratificação permitida no questionário original da PNAD (nunca, três ou mais anos, menos de três anos); número de consultas médicas nos últimos 12 meses e cobertura por plano de saúde (plano de saúde particular, de empresa ou órgão público) 8 .

\section{Análise dos dados}

As características das mulheres que haviam realizado a mamografia há dois anos ou menos foram comparadas às daquelas que haviam realizado $o$ exame há mais tempo ou nunca haviam realizado uma mamografia. A análise foi realizada separadamente para a faixa etária de 50-59 e 60-69 anos.

A análise dos dados foi baseada em OR brutos e ajustados. Os OR foram estimados por meio de regressão logística univariada e multivariada 10. A inclusão das variáveis no modelo logístico obedeceu a uma ordem hierárquica, incluindo inicialmente as características sócio-demográficas, a seguir os indicadores de condições de saúde e, finalmente, os indicadores de usos de serviços de saúde. Aquelas que permaneceram significativamente ( $p<0,05)$ associadas à realização da mamografia foram incluídas no modelo seguinte e assim sucessivamente.

As análises foram realizadas utilizando-se os procedimentos do programa Stata (Stata Corp., College Station, Estados Unidos) para inquéritos populacionais, incorporando os pesos individuais (inverso da fração amostral) e o efeito do delineamento do estudo.

\section{Resultados}

Participaram do presente trabalho $16.570 \mathrm{mu}-$ lheres na faixa etária de 50-59 anos e 10.722 mulheres na faixa etária de 60-69 anos. As médias de idade foram 58 e 64 anos, respectivamente.

A distribuição da realização da mamografia aos 50-69 anos de idade foi a seguinte: $42,5 \%$ haviam realizado o exame há dois anos ou menos, $8,2 \%$ há três anos ou mais e 49,3\% em nenhuma época da vida. A distribuição correspondente nos dois grupos etários foi 46,3\%; 7,6\% e 46,1\% entre as mais jovens e $36,9 \% ; 9,1 \%$ e $54 \%$ entre as mais velhas $(\mathrm{p}<0,001)$.

Na Tabela 1, estão apresentados os resultados da análise univariada das características sócio-demográficas associadas à realização da mamografia há dois anos ou menos, segundo a faixa etária. Em ambos os grupos, associações positivas e significantes foram encontradas para condição urbana ou rural do domicílio, macrorregião de residência, escolaridade e renda familiar per capita. Morar só apresentou associação estatisticamente significante com a realização da mamografia entre as mais jovens, mas não entre as mais velhas.

Todos os indicadores das condições de saúde considerados neste trabalho apresentaram, na análise univariada, associações negativas e significantes com a realização do exame em ambos os grupos etários, como pode ser visto na Tabela 2. Associações positivas e significantes foram observadas para todos os indicadores de usos de serviços de saúde, tanto na faixa de 50-59 quanto na de 60-69 anos de idade (Tabela 3).

Na Tabela 4, estão apresentados os resultados finais da análise multivariada das características sócio-demográficas associadas à realização da mamografia, segundo a faixa etária. Em ambas as faixas etárias, foram observadas associações positivas e significantes com a localização do domicílio (urbana em relação à rural), 
Resultados da análise univariada da associação entre características sócio-demográficas e realização da mamografia há dois anos ou menos entre mulheres com 50-59 anos e 60-69 anos de idade. Brasil: Pesquisa Nacional por Amostra de Domicílios, 20036

\begin{tabular}{|c|c|c|c|c|c|c|}
\hline \multirow[t]{3}{*}{ Características sócio-demográficas } & \multicolumn{3}{|c|}{ 50-59 anos } & \multicolumn{3}{|c|}{$60-69$ anos } \\
\hline & \multicolumn{2}{|c|}{ Mamografia } & \multirow[t]{2}{*}{ OR (IC95\%) } & \multicolumn{2}{|c|}{ Mamografia } & \multirow[t]{2}{*}{ OR (IC95\%) } \\
\hline & Sim & Não & & Sim & Não & \\
\hline \multicolumn{7}{|l|}{ Condição do domicílio } \\
\hline Rural & 19,0 & 81,0 & 1,00 & 12,9 & 87,1 & 1,00 \\
\hline Urbana & 50,9 & 49,1 & $4,52(3,48-5,88)$ & 41,1 & 58,9 & $4,41(3,29-5,90)$ \\
\hline \multicolumn{7}{|l|}{ Macrorregião de residência } \\
\hline Norte & 30,2 & 69,8 & 1,00 & 21,4 & 78,6 & 1,00 \\
\hline Nordeste & 30,0 & 70,0 & $0,98(0,58-1,66)$ & 23,0 & 77,0 & $1,25(0,72-2,14)$ \\
\hline Centro-Oeste & 48,5 & 51,5 & $2,14(1,35-3,39)$ & 36,3 & 63,7 & $2,13(1,31-3,46)$ \\
\hline Sudeste & 55,5 & 44,5 & $2,92(1,84-4,62)$ & 46,4 & 53,6 & $3,26(1,96-5,43)$ \\
\hline Sul & 46,1 & 53,9 & $1,94(1,17-3,20)$ & 34,8 & 65,2 & $2,12(1,22-3,69)$ \\
\hline \multicolumn{7}{|l|}{ Mora só } \\
\hline Não & 46,0 & 54,0 & 1,00 & 36,5 & 63,5 & 1,00 \\
\hline $\operatorname{Sim}$ & 50,9 & 49,1 & $1,16(1,03-1,32)$ & 40,0 & 60,0 & $1,15(0,93-1,42)$ \\
\hline \multicolumn{7}{|l|}{ Anos completos de escolaridade } \\
\hline$<3$ & 28,5 & 71,5 & 1,00 & 25,0 & 75,0 & 1,00 \\
\hline $4-7$ & 48,1 & 51,9 & $2,28(1,95-2,67)$ & 41,4 & 58,6 & $2,16(1,80-2,58)$ \\
\hline$\geq 8$ & 68,2 & 31,8 & $5,50(4,41-6,85)$ & 65,6 & 34,4 & $5,95(4,87-7,26)$ \\
\hline \multicolumn{7}{|l|}{ Renda familiar per capita (em quintis) * } \\
\hline 1으 & 21,8 & 78,2 & 1,00 & 18,2 & 81,8 & 1,00 \\
\hline 2o & 33,5 & 66,5 & $1,72(1,50-1,98)$ & 24,4 & 75,6 & $1,29(1,03-1,61)$ \\
\hline 3으 & 41,8 & 58,2 & $2,41(2,04-2,84)$ & 29,7 & 70,3 & $1,55(1,22-1,96)$ \\
\hline 4으 & 57,8 & 42,2 & $4,49(3,68-5,49)$ & 42,3 & 57,7 & $2,75(2,16-3,52)$ \\
\hline 5으 & 74,9 & 25,1 & $10,42(8,10-13,41)$ & 66,7 & 33,3 & $7,73(5,68-10,52)$ \\
\hline
\end{tabular}

* Distribuição em quintis do salário mínimo da época ( $\mathrm{R}$ 240,00).

a macrorregião de residência (regiões Nordeste, Centro-Oeste, Sudeste e Sul, em comparação à região Norte), a escolaridade (4-7 e $\geq 8$ anos, em comparação à escolaridade mais baixa) e a renda

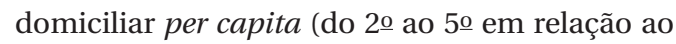
1o quintil).

Os resultados finais da análise multivariada dos indicadores das condições de saúde e de usos de serviços de saúde associados à mamografia estão apresentados na Tabela 5. As condições de saúde não apresentaram associações independentes com a realização da mamografia entre as mulheres mais jovens. Entre as mais velhas, a pior percepção da saúde (razoável e ruim/muito ruim) apresentou associação negativa com esse exame. Nas duas faixas etárias, foi observada associação positiva entre realização da mamografia e exame de Papanicolau (há três anos ou mais e há menos de três anos), número de consultas a um médico nos últimos 12 meses (1, 2 e 3 ou +) e cobertura por plano de saúde.

\section{Discussão}

A realização da mamografia, nos dois anos precedentes, entre mulheres brasileiras com 50-59 (46\%) e 60-69 anos de idade (37\%), está muito abaixo da meta de $70 \%$ estabelecida no documento Healthy People $2010^{3}$. Além disso, observou-se uma alta prevalência de mulheres que, ao longo da vida, jamais tiveram uma mamografia realizada, tanto na faixa etária mais jovem (46\%) quanto na mais velha (49\%).

Diversos estudos têm mostrado que a situação sócio-econômica e/ou a escolaridade estão entre os fatores mais fortemente associados à realização da mamografia 7,11,12,13,14. Os resultados do presente trabalho confirmam essas observações. A renda domiciliar e a escolaridade apresentaram forte associação com a realização do exame. Essas associações foram graduadas verificando-se um aumento progressivo da realização da mamografia com aumento da escolaridade, assim como com o aumento da renda familiar 
Resultados da análise univariada da associação entre indicadores das condições de saúde e realização da mamografia há dois anos ou menos entre mulheres com 50-59 anos e 60-69 anos de idade. Brasil: Pesquisa Nacional por Amostra de Domicílios, 20036.

\begin{tabular}{|c|c|c|c|c|c|c|}
\hline \multirow[t]{3}{*}{ Condições de saúde } & \multicolumn{3}{|c|}{$50-59$ anos } & \multicolumn{3}{|c|}{$60-69$ anos } \\
\hline & \multicolumn{2}{|c|}{ Mamografia } & \multirow[t]{2}{*}{ OR (IC95\%) } & \multicolumn{2}{|c|}{ Mamografia } & \multirow[t]{2}{*}{ OR (IC95\%) } \\
\hline & Sim & Não & & Sim & Não & \\
\hline \multicolumn{7}{|c|}{ Percepção da saúde no domicílio } \\
\hline Muito boa/Boa & 51,6 & 48,4 & 1,00 & 43,1 & 56,9 & 1,00 \\
\hline Regular & 41,2 & 58,8 & $0,65(0,60-0,61)$ & 33,5 & 66,5 & $0,70(0,61-0,80)$ \\
\hline Ruim/Muito ruim & 36,6 & 63,4 & $0,52(0,46-0,58)$ & 25,9 & 74,1 & $0,42(0,34-0,52)$ \\
\hline \multicolumn{7}{|l|}{ Número de doenças crônicas } \\
\hline 0 & 46,7 & 53,3 & 1,00 & 36,4 & 63,6 & 1,00 \\
\hline 1 & 45,6 & 54,4 & $0,98(0,92-1,05)$ & 37,9 & 62,1 & $0,95(0,79-1,14)$ \\
\hline 2 & 46,4 & 53,6 & $0,96(0,89-1,04)$ & 35,6 & 64,4 & $0,91(0,73-1,13)$ \\
\hline$\geq 3$ & 47,3 & 52,7 & $0,99(0,87-1,14)$ & 37,6 & 62,4 & $0,97(0,75-1,26)$ \\
\hline \multirow{2}{*}{\multicolumn{7}{|c|}{$\begin{array}{l}\text { Grau de dificuldade para realizar } \\
\text { atividades da vida diária * }\end{array}$}} \\
\hline & & & & & & \\
\hline Nenhum & 47,0 & 53,0 & 1,00 & 38,1 & 61,9 & 1,00 \\
\hline Pequeno & 35,7 & 64,3 & $0,62(0,55-0,71)$ & 27,5 & 72,5 & $0,59(0,46-0,74)$ \\
\hline Grande/Não consegue & 31,7 & 68,3 & $0,50(0,39-0,65)$ & 21,0 & 79,0 & $0,37(0,25-0,56)$ \\
\hline
\end{tabular}

* Atividades da vida diária (pelo menos uma entre alimentar-se, tomar banho e/ou ir ao banheiro).

Tabela 3

Resultados da análise univariada da associação entre o uso de serviços de saúde e realização da mamografia há dois anos ou menos entre mulheres com 50-59 anos e 60-69 anos de idade. Brasil: Pesquisa Nacional por Amostra de Domicílios, 20036.

\begin{tabular}{|c|c|c|c|c|c|c|}
\hline \multirow[t]{3}{*}{ Uso de serviços de saúde } & \multicolumn{3}{|c|}{$50-59$ anos } & \multicolumn{3}{|c|}{$60-69$ anos } \\
\hline & \multicolumn{2}{|c|}{ Mamografia } & \multirow[t]{2}{*}{ OR (IC95\%) } & \multicolumn{2}{|c|}{ Mamografia } & \multirow[t]{2}{*}{ OR (IC95\%) } \\
\hline & Sim & Não & & Sim & Não & \\
\hline \multicolumn{7}{|l|}{ Exame Papanicolau (anos) } \\
\hline Nunca & 5,2 & 94,8 & 1,00 & 3,5 & 96,5 & 1,00 \\
\hline Três anos ou menos & 15,9 & 84,1 & $3,46(2,74-4,37)$ & 12,9 & 87,1 & $4,05(2,99-5,49)$ \\
\hline Menos de três anos & 64,7 & 35,3 & $33,54(28,23-39,85)$ & 61,7 & 38,3 & $44,00(34,50-56,13)$ \\
\hline \multicolumn{7}{|c|}{ Número de consultas médicas } \\
\hline \multicolumn{7}{|l|}{ nos últimos 12 meses } \\
\hline 0 & 25,5 & 74,5 & 1,00 & 17,7 & 82,3 & 1,00 \\
\hline 1 & 42,3 & 57,7 & $2,10(1,72-2,56)$ & 31,9 & 68,1 & $2,52(1,69-3,78)$ \\
\hline 2 & 48,2 & 51,8 & $2,73(2,23-3,34)$ & 38,8 & 61,2 & $3,15(2,26-4,39)$ \\
\hline$\geq 3$ & 54,7 & 45,3 & $3,41(2,80-4,15)$ & 43,3 & 56,7 & $3,96(2,97-5,27)$ \\
\hline \multicolumn{7}{|c|}{ Cobertura por plano de saúde } \\
\hline Não & 34,9 & 65,1 & 1,00 & 25,4 & 74,6 & 1,00 \\
\hline Sim & 71,0 & 29,0 & $4,74(4,08-5,22)$ & 62,5 & 37,5 & $4,41(3,58-5,44)$ \\
\hline
\end{tabular}


Resultados finais da análise multivariada das características sócio-demográficas associadas à realização da mamografia há dois anos ou menos entre mulheres com 50-59 anos e 60-69 anos de idade. Brasil: Pesquisa Nacional por Amostra de Domicílios, 20036

\begin{tabular}{|c|c|c|}
\hline \multirow[t]{2}{*}{ Características sócio-demográficas } & \multicolumn{2}{|c|}{ OR ajustado (IC95\%) } \\
\hline & $50-59$ anos & $60-69$ anos \\
\hline \multicolumn{3}{|l|}{ Condição do domicílio } \\
\hline Rural & 1,00 & 1,00 \\
\hline Urbana & $2,09(1,65-2,65)$ & $2,31(1,92-2,79)$ \\
\hline \multicolumn{3}{|l|}{ Macrorregião de residência } \\
\hline Norte & 1,00 & 1,00 \\
\hline Nordeste & $1,74(1,23-2,48)$ & $1,64(1,08-2,49)$ \\
\hline Centro-Oeste & $2,36(1,68-3,31)$ & $1,83(1,21-2,75)$ \\
\hline Sudeste & $2,85(2,09-3,89)$ & $2,78(1,84-4,18)$ \\
\hline Sul & $2,02(1,39-2,95)$ & $1,76(1,12-2,78)$ \\
\hline \multicolumn{3}{|l|}{ Anos completos de escolaridade } \\
\hline$<3$ & 1,00 & 1,00 \\
\hline $4-7$ & $1,39(1,23-1,56)$ & $1,14(1,01-1,29)$ \\
\hline$\geq 8$ & $1,86(1,56-2,22)$ & $1,97(1,65-2,36)$ \\
\hline \multicolumn{3}{|l|}{ Renda familiar per capita (em quintis) * } \\
\hline 1 으 & 1,00 & 1,00 \\
\hline 2으 & $1,34(1,10-1,63)$ & $1,20(1,00-1,43)$ \\
\hline 3으 & $1,48(1,18-1,85)$ & $1,45(1,21-1,72)$ \\
\hline 4으 & $2,18(1,77-2,69)$ & $1,52(1,26-1,84)$ \\
\hline 5으 & $3,18(2,40-4,23)$ & $2,27(1,83-2,81)$ \\
\hline
\end{tabular}

Odds ratio (OR) ajustado pelo método de regressão logística por todas as variáveis listadas nessa tabela e pelas variáveis listadas na Tabela 5 correspondentes às condições de saúde e uso de serviços de saúde (modelo final incluiu 14.515 mulheres com 50-59 anos e 9.995 mulheres com 60-69 anos).

* Distribuição em quintis do salário mínimo da época ( $R \$ 240,00)$.

per capita, independente de todos as demais variáveis consideradas no trabalho. Associações fortes e independentes foram também observadas para a condição rural/urbana do domicílio e para a macrorregião de residência, verificando-se a menor realização do exame entre os residentes na zona rural e os residentes nas regiões Norte e Nordeste do país.

Alguns estudos têm mostrado que a realização da mamografia é menos freqüente entre mulheres com piores condições de saúde 14,15,16. No presente trabalho, foram considerados três indicadores da condição de saúde: percepção da saúde, número de doenças crônicas e grau de dificuldade para realizar atividades da vida diária. O primeiro é um dos indicadores mais utilizados em pesquisas gerontológicas, uma vez que tem sido demonstrado que a pior percepção da saúde é um preditor independente da mortalidade de outros adventos adversos 17,18. O segundo e o terceiro indicadores expressam, respectivamente, co-morbidade e capacidade funcional.
A análise não ajustada mostrou, em ambas as faixas etárias, que havia uma associação negativa entre realização da mamografia e os três indicadores acima mencionados. Entretanto, após ajustamentos por variáveis de confusão, todas as associações desapareceram na faixa etária mais jovem. Na faixa etária superior, somente pior percepção da saúde permaneceu independentemente associada à realização da mamografia, sugerindo que a importância do exame possa estar sendo minimizada diante de outros problemas de saúde.

A recomendação médica é o preditor mais forte da realização da mamografia ${ }^{19}$. Dessa forma, o contato profissional com um médico (ou um indicador desse contato) é freqüentemente descrito como associado à realização do exame $7,12,13,14,15,16$. Os resultados deste trabalho são coerentes com essas observações, uma vez que, em ambas as faixas etárias, o número de consultas médicas apresentou associação graduada com a realização da mamografia. Além disso, verificou- 
Resultados finais da análise multivariada das condições de saúde e uso de serviços de saúde associados à realização da mamografia há dois anos ou menos entre mulheres com 50-59 anos e 60-69 anos de idade. Brasil: Pesquisa Nacional por Amostra de Domicílios, 20036

\begin{tabular}{|c|c|c|}
\hline & \multicolumn{2}{|c|}{ OR ajustado (IC95\%) } \\
\hline & $50-59$ anos & $60-69$ anos \\
\hline \multicolumn{3}{|l|}{ Condições de saúde } \\
\hline \multicolumn{3}{|l|}{ Percepção da saúde } \\
\hline Muito boa/Boa & 1,00 & 1,00 \\
\hline Regular & NS & $0,83(0,69-1,00)$ \\
\hline Ruim/Muito ruim & NS & $0,78(0,63-0,98)$ \\
\hline \multicolumn{3}{|l|}{ Uso de serviços de saúde } \\
\hline \multicolumn{3}{|l|}{ Exame Papanicolau (anos) } \\
\hline Nunca & 1,00 & 1,00 \\
\hline Três anos ou menos & $2,48(1,94-3,16)$ & $2,58(1,91-3,52)$ \\
\hline Menos de três anos & $24,12(20,13-28,89)$ & $29,55(23,61-36,97)$ \\
\hline \multicolumn{3}{|c|}{ Número de consultas médicas } \\
\hline \multicolumn{3}{|c|}{ nos últimos 12 meses } \\
\hline 0 & 1,00 & 1,00 \\
\hline 1 & $1,31(1,14-1,52)$ & $1,50(1,15-1,96)$ \\
\hline 2 & $1,70(1,34-2,14)$ & $2,05(1,57-2,68)$ \\
\hline$\geq 3$ & $2,11(1,75-2,54)$ & $2,20(1,67-2,88)$ \\
\hline \multicolumn{3}{|c|}{ Cobertura por plano de saúde } \\
\hline Não & 1,00 & 1,00 \\
\hline Sim & $1,68(1,53-1,84)$ & $2,04(1,82-2,29)$ \\
\hline
\end{tabular}

Odds ratio (OR) ajustado pelo método de regressão logística por todas as variáveis listadas nessa tabela e pelas variáveis listadas na Tabela 4 correspondentes às características sócio-demográficas (modelo final incluiu 14.515 mulheres com 50-59 anos e 9.995 mulheres com 60-69 anos).

NS: não significante.

se que o exame de Papanicolau (um indicador de consulta a um ginecologista) era a variável mais fortemente associada à realização da mamografia.

Estudos anteriormente conduzidos em diferentes capitais brasileiras mostram que a realização da mamografia é mais freqüente entre mulheres que possuem cobertura por plano privado de saúde 4,6. Os resultados do presente trabalho confirmam essas observações e acrescentam por mostrar que a associação permanece mesmo após ajustamentos por situação sócio-econômica (renda e escolaridade), local de residência (urbana/rural e macrorregião), número de consultas médicas e realização do exame de Papanicolau. É possível que a maior freqüência da mamografia entre mulheres filiadas a plano de saúde seja devido à solicitação mais freqüente do exame, à maior disponibilidade de aparelhos para a sua realização ou a ambos os fatores.

Dois estudos de base populacional realizados no Brasil 6,7 e um estudo de mulheres filiadas a plano de saúde nos Estados Unidos 11 mostraram que a realização da mamografia era mais freqüente entre as mais jovens do que entre as mais velhas. Resultados semelhantes foram obtidos no presente trabalho. A realização menos freqüente da mamografia na faixa etária de 60-69 anos, em comparação à faixa de 50-59 anos, não se justifica, uma vez que a incidência de câncer de mama aumenta com a idade, e a efetividade desse exame para a prevenção da mortalidade por esse câncer está bem estabelecida em ambos os grupos etários. As explicações para a menor realização da mamografia entre idosas não são evidentes, uma vez que - no presente trabalho os fatores associados à realização do exame não diferiram nas duas faixas etárias consideradas, exceto pela percepção da saúde, como anteriormente comentado. Uma explicação alternativa é um efeito de coorte na disseminação dessa prática, que estaria aumentando nas coortes mais jovens. 
O presente trabalho tem as limitações inerentes ao uso de dados secundários. A principal dessas limitações é a inexistência, na base de dados da PNAD, de informações sobre outras variáveis importantes para o entendimento dos fatores que determinam a realização da mamografia. Esses fatores incluem uso de outros serviços preventivos de saúde 11,14, história médica de doenças mamárias e outras condições ginecológicas 12, suporte social e eventos negativos de vida 20 , conhecimento de fatores de risco 21 , entre outros. Por outro lado, a utilização da base de dados da PNAD para determinar a distribuição e os fatores associados à mamografia tem a vantagem de per-

\section{Resumo}

O presente trabalho foi desenvolvido utilizando dados da Pesquisa Nacional por Amostra de Domicílios de 2003 (PNAD 2003), com o objetivo de estimar a prevalência e os fatores associados à realização da mamografia entre mulheres brasileiras com 50-59 e 60-69 anos de idade. Participaram do estudo 16.570 e 10.722 mulheres nas respectivas faixas etárias. Quarenta e três por cento das participantes com 50-69 anos haviam realizado uma mamografia nos últimos dois anos. Essa prevalência foi mais baixa na faixa etária superior (37\%) em comparação à inferior (46\%). Em ambas as faixas etárias, a realização da mamografia apresentou associações positivas e independentes com a localização urbana do domicílio, a macrorregião de residência, a escolaridade, a renda domiciliar, o exame de Papanicolau, o número de consultas médicas e a cobertura por plano de saúde. Esses resultados mostram que a cobertura da mamografia ainda é baixa entre mulheres brasileiras, sobretudo entre as mais velhas, $e$ que existem profundas desigualdades associadas à sua realização. Recomenda-se a condução de investigações mais profundas que permitam um melhor entendimento dessas desigualdades, visando a sua superação.

Mamografia; Saúde do Idoso; Saúde da Mulher; Mulheres mitir a generalização do resultados para a população feminina brasileira na faixa etária de 50-69 anos (exceto interior da Região Norte).

Em resumo, os resultados deste trabalho mostraram que existem profundas desigualdades sociais, regionais e etárias na realização da mamografia entre mulheres brasileiras. Essas desigualdades são inaceitáveis, uma vez que a mamografia é um instrumento da Saúde Pública, com efetividade comprovada. São necessárias investigações com base em dados primários para um melhor entendimento dessas desigualdades, visando a sua superação.

\section{Colaboradores}

M. F. Lima-Costa delineou o estudo, analisou os dados e redigiu o manuscrito. D. L. Matos preparou o banco de dados, participou da análise dos dados e da revisão crítica do manuscrito.

\section{Agradecimentos}

Este trabalho foi desenvolvido como parte das atividades do Núcleo de Estudos em Saúde Pública e Envelhecimento, Centro de Pesquisas René Rachou, Fundação Oswaldo Cruz/Universidade Federal de Minas Gerais com o Centro Colaborador em Saúde do Idoso, Secretaria de Vigilância à Saúde, Ministério da Saúde. A pesquisa contou com recursos do Ministério da Saúde. Maria Fernanda Lima-Costa é pesquisadora bolsista do Conselho Nacional de Desenvolvimento Científico e Tecnológico. 


\section{Referências}

1. Departamento de Informação e Informática do SUS. Sistema de Informações sobre Mortalidade (SIM), 1996-2000 [CD-ROM]. Brasília: Departamento de Informação e Informática do SUS; 2000.

2. Lima-Costa MF, Peixoto SV, Giatti L. Tendências da mortalidade entre idosos brasileiros (1980-2000). Epidemiol Serv Saúde 2004; 13:217-28.

3. Centers for Disease Control and Prevention. Healthy people 2010. http://www.healthypeople. gov (acessado em 13/Out/2005).

4. Ministério da Saúde/Instituto Nacional de Câncer/ Secretaria de Vigilância em Saúde. Inquérito domiciliar sobre comportamentos de risco e morbidade referida de doenças e agravos não transmissíveis: Brasil, 15 capitais e Distrito Federal, 2002-2003. Rio de Janeiro: Instituto Nacional de Câncer; 2004.

5. Lima-Costa MF. Influência da idade e da escolaridade no uso de serviços preventivos de saúde - inquérito de saúde da Região Metropolitana de Belo Horizonte, Minas Gerais, Brasil. Epidemiol Serv Saúde 2004; 13:209-15.

6. Lima-Costa MF. Estilos de vida e uso de serviços preventivos de saúde entre adultos filiados ou não a plano privado de saúde (inquérito de saúde de Belo Horizonte). Ciênc Saúde Coletiva 2004; 9:85764.

7. Sclowitz ML, Menezes AMB, Gigante DP, Tessaro S. Condutas na prevenção secundária do câncer de mama e fatores associados. Rev Saúde Pública 2005; 39:340-9.

8. Instituto Brasileiro de Geografia e Estatística. Pesquisa Nacional por Amostra de Domicílios, 2003. Rio de Janeiro: Instituto Brasileiro de Geografia e Estatística; 2003.

9. Lima-Costa MF, Peixoto SV, Matos DL, Firmo JOA, Uchôa E. A influência de respondente substituto na percepção da saúde de idosos: um estudo baseado na Pesquisa Nacional por Amostra de Domicílios $(1998,2003)$ e na coorte de Bambuí, Minas Gerais, Brasil. Cad Saúde Pública; no prelo.

10. Hosmer Jr. DW, Lemenshow S. Applied logistic regression. New York: John Wiley and Sons; 1989.
11. Morales LS, Rogowski J, Freedman VA, Wickstrom SL, Adams JL, Escarce JJ. Sociodemographic differences in use of preventive services by women enrolled in Medicare + Choice plans. Prev Med 2004; 39:738-45.

12. Otero-Sabogal R, Owens D, Canchola J, Golding JM, Tabnak F, Fox P. Mammography rescreening among women of diverse ethnicities: patient, provider, and health care system factors. J Health Care Poor Underserved 2004; 15:390-412.

13. David MM, Ko L, Prudent N, Green EH, Posner MA, Freund KM. Mammography use. J Natl Med Assoc 2005; 97:253-61.

14. Barret K, Legg J. Demographic and health factors associated with mammography utilization. Am J Health Promot 2005; 19:401-5.

15. Achat H, Close G, Taylor R. Who has regular mammograms? Effect of knowledge, beliefs, socioeconomic status, and health-related factors. Prev Med 2005; 41:312-20.

16. Greene AL, Torio CM, Klassen AC. Measuring sustained mammography use by urban AfricanAmerican women. J Community Health 2005; 30:235-51.

17. Idler EL, Kasl SV. Self-rating of health: do they also predict change in functional ability? J Gerontol B Psychol Sci Soc Sci 1995; 50:S344-53.

18. Grundy E, Sloggett A. Health inequalities in the older population: the role of personal capital, social resources and socio-ecomomic circumstances. Soc Sci Med 2003; 56: 935-47.

19. Rauscher GH, Hawley ST, Earp JA. Baseline predictors of initiation vs. maintenance of regular mamography use among rural women. Prev Med 2005; 40:822-30.

20. Messina CR, Lane DS, Glanz K, West DS, Taylor V, Frishman W, et al. Relationship of social support and social burden to repeated breast cancer screening in the women's health initiative. Health Psychol 2004; 23:582-94.

21. Ahmed NU, Fort JG, Elzey JD, Belay Y. Empowering factors for regular mammography screening in under-served populations: pilot survey results in Tennessee. Ethn Dis 2005; 15:387-94.

Recebido em 24/Abr/2006

Versão final reapresentada em 22/Dez/2006

Aprovado em 04/Jan/2007 